Aim To examine trends in hospitalisations for TBI in adults aged 65 years and over, in New South Wales, Australia.

Methods TBI cases from 1998 to 2011 were identified from hospitalisation data for all public and private hospitals in NSW. Age-standardised admission rates were calculated. Negative binomial regression modelling was used to examine trends over time.

Results There were 12564 hospitalisations for TBI over the 13 year study period. Hospitalisation rates increased by $7.2 \%$ (95\% CI 6.4 to $8.0, p<0.0001)$ per year from $19.3 / 100000$ to $72.2 / 100000$. One third of all hospitalisations were for adults aged 85 years and over. Traumatic subdural haemorrhage (42.9\%), concussive injury (24.1\%) and traumatic subarachnoid haemorrhage (12.7\%) were the most common type of injury. Falls were the most common cause of TBI (82.9\%). Rates of fall-related TBI increased by $8.4 \%$ (95\% CI 7.5 to $9.3, p<0.001)$ per year, whilst non-fall related TBI increased by $2.1 \%$ ( $95 \%$ CI 0.9 to $3.3, \mathrm{p}<0.0001)$ per year. $13 \%$ of hospitalisations resulted in death, and the majority occurred in those who sustained a traumatic subdural haemorrhage.

Significance The dramatic increase in hospitalised TBI is being predominantly driven by falls in the oldest old, resulting in intracranial haemorrhages. The cause for this is uncertain but may relate to the increasing use of anticoagulant therapies. More research is required to better understand the observed patterns.

\section{2 \\ CHARACTERISTICS, CAUSES AND CONSEQUENCES OF HOSPITALISED TRAUMATIC BRAIN INJURY IN OLDER ADULTS}

doi:10.1136/injuryprev-2012-040590e.12

Harvey Lara, Close Jacqueline. Falls and Injury Prevention Group, Neuroscience Research Australia, Barker Street, Randwick, NSW, 2031 Australia

Background Traumatic brain injury (TBI) is an important cause of injury-related hospitalisation, disability and death worldwide, and is of particular concern in the older population where an acute insult to the brain can end independent living. 